\title{
A ROBUST APPROACH FOR REDUCING PEAK TO AVERAGE POWER RATIO IN OFDM SYSTEM
}

\author{
Rizwan Ahmed Mailk ${ }^{1}$, Ruchi Singla ${ }^{2}$ \\ ${ }^{I}$ Student M.Tech (Scholar), Chandigarh Engineering College, Landran \\ ${ }^{2}$ Head of ECE, Chandigarh Engineering College, Landran \\ [rizwanmalik520562, cecm.ece.rps]@gmail.com
}

\begin{abstract}
The usage of orthogonal frequency division multiplexing (OFDM) approach arrange for better key for the overhead mentioned problems. OFDM approach issues the information over a huge number of exporters that are spaced separately at exact frequencies. So the advantages of OFDM are great spectral efficiency, toughness of RF interference \& minor various path distortions. Generally in OFDM systems there are lot of chances of inter-symbol interferences(ISI), inter carrier interference(ICI) and band limited channel due to multipath fading's which results in huge path delays and less effective recovery of the signal at the receiver end. These can be due to the PAPR effects which should be minimized. For this reason band limited signal often used pulse shaping technique and equalization. In this paper, pulse shaping of multicarrier signal is proposed by using raised cosine pulse $(R C)$. The result achieved by simulation of OFDM system by the use of pulse shaping indicates that the performance of OFDM is better without using pulse shaping and equalization at the receiving side.
\end{abstract}

Keyword:-OFDM Transmitter \& Receiver, Orthogonal, Multi-Path Effect, Digital Audio Broadcasting $* * *$

\section{INTRODUCTION}

OFDM is a combination of variation and multiplexing. Multiplexing usually refers to independent signals, individuals produced by numerous bases. In OFDM the queries of multiplexing is functional to self-determining signals but these self-governing indicators are a sub-set of the 1 main signal. Fig. 1 shows the subcarriers.

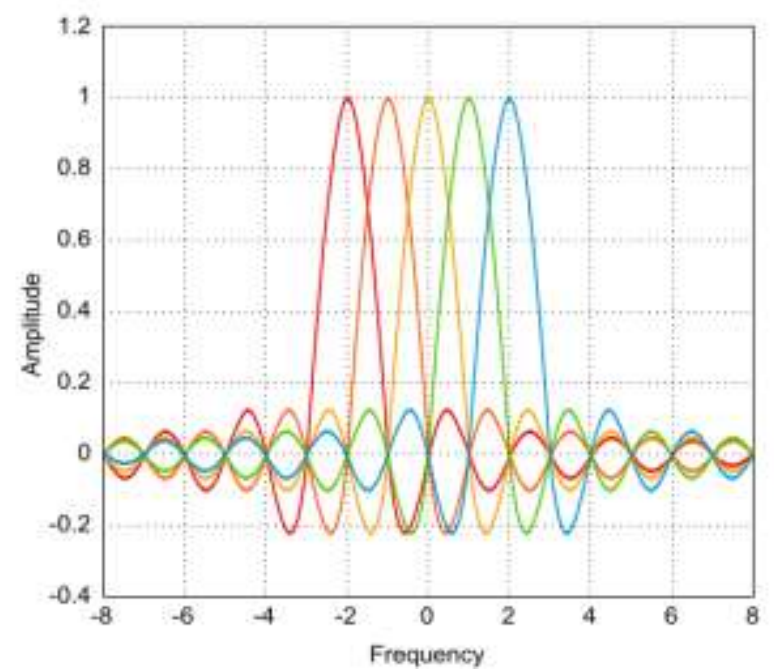

Fig: 1. OFDM Frequency Carrier [1]

In OFDM the signal themself is main divided into selfgoverning networks, modulated by data \& then remultiplexed to create the OFDM carrier. OFDM is a superior case of Frequency Division Multiplexing(FDM). In an OFDM system, a huge number of orthogonal, overlying, thin band sub-carriers are transferred in parallel. The parting of the sub-carriers is such that there is a very compact spectral utilization [1].

\subsection{OFDM Transmitter}

The Fig. 2 shows the OFDM transmitter consisting of the following blocks:

1) Input Sampler:- First block in the transmitter section of OFDM system is input sampler. Data is fed into the block as serial, and two bit IQ is the output. The output is the combination of symbols, in which everyone containing two bits. So the sampler block combines two bits.

2) Symbol Mapper:- The output from the sampler is applied as input to the symbol mapper. QPSK modulator is used in symbol mapper. The two bit data given to the modulator divide into I and Q (imaginary and quadrature) symbols which is two bits. Based on the constellation diagram these signals are generated. There are four phases in QPSK, in which each two bit symbol is assigned to a phase, and the difference between them is 90 degree. Based on the constellation diagram of QPSK the coding is done. 


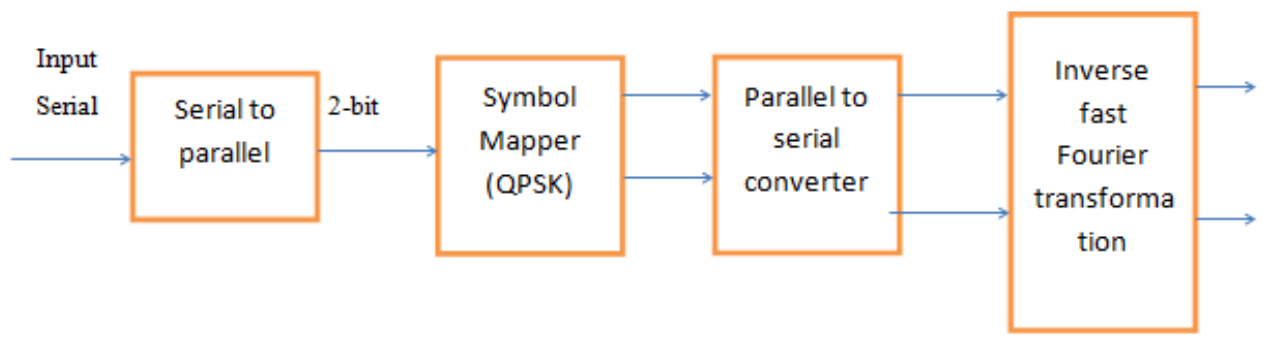

Fig: 2. OFDM Transmitter

3) Serial input parallel output (SIPO):-The symbol mapper output act as input for this block. In this block data is converted from serial to parallel form. Serial input parallel output is a two 8 registers $(0-7)$ array. In the seventh array the serial input is fed and data is shifted to the above register for every clock cycle. Data in the array is forwarded after 8 clock cycles. SIPO contains eight register of real data and eight of imaginary data.

4) Inverse fast Fourier transform (IFFT):- the most important module in OFDM system is IFFT. The output from the serial input parallel output is given to the IFFT block. We require two modules in this system, one is for the real and one is for imaginary. The IFFT converts signal from frequency domain into time domain. Through the transmitter the time domain values transmitted in the form of OFDM signals. IFFT is carried out in steps in this system. Firstly exchange real and imaginary parts, then perform FFT, exchange the real and the imaginary parts, and in last divide with N, i.e number of inputs fed, here it is eight [2].

\subsection{OFDM Receiver}

The OFDM Receiver is shown in the Fig.3.FFT preferred over DFT for demodulation as it reduced the processing time and hardware used. FFT computes DFT with a countless decrease in the quantity of processes, parting several existent dismissals in the direct control of DFT. At the decoder, a charted symbol (point) of the communicated gathering may have changed due to the preservative noise in the infrastructures channel; goal is adjustment in the sample time at the receiver, or numerous other undesirable causes [3].

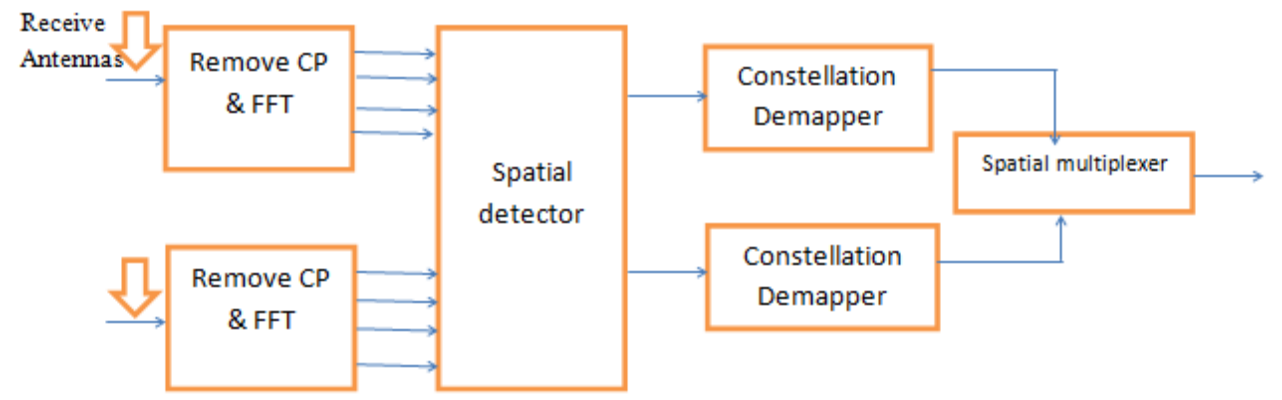

Fig: 3. OFDM receiver

Table 1: Advantages\&Disadvantages [4].

\begin{tabular}{|l|l|}
\hline Advantages of OFDM & Disadvantages of OFDM \\
\hline $\begin{array}{l}\text { 1. OFDM varieties inventive application of the spectrum } \\
\text { by overlying. By separating the channel into thinband flat } \\
\text { fading sub channels, OFDM is added impervious to } \\
\text { frequency selective disappearing than solitary carrier } \\
\text { systems. }\end{array}$ & $\begin{array}{l}\text { 1. The OFDM sign has a sound like breadth with a very } \\
\text { great lively range; hence it involves RF power amplifiers } \\
\text { with a great peak to average power ratio. }\end{array}$ \\
\hline $\begin{array}{l}\text { 2. It can easily adapt to plain channel circumstances } \\
\text { without multifarious time-domain equalization. }\end{array}$ & $\begin{array}{l}\text { 2. It is additional sensitive to carrier frequency } \\
\text { counterweight \& drift than single carrier systems are due } \\
\text { to leak of the DFT (Discrete Fourier transform). }\end{array}$ \\
\hline $\begin{array}{l}\text { 3. It reduces inter symbol interference complete use of a } \\
\text { cyclic prefix \& fading produced by multi-path broadcast } \\
\text { [11]. }\end{array}$ & \begin{tabular}{l} 
3. It is sensitive to Doppler shift. \\
\hline $\begin{array}{l}\text { 4. Using appropriate channel coding \& encoding lost } \\
\text { signal can be recoverd. }\end{array}$
\end{tabular} \\
\hline $\begin{array}{l}\text { 5. Channel equalization develops greener than by using } \\
\text { adaptive equalization methods with single carrier systems. }\end{array}$ & 5. It gives loss of competence caused by cyclic prefix. \\
\hline
\end{tabular}




\section{ORTHOGONALITY OF SUB-CARRIERS}

\subsection{IFFT (Inverse Fast Fourier Transform)}

Encode: Sample of frequency-domain into time-domain sample

$$
\mathrm{x}(\mathrm{t})=\sum_{k=-N / 2}^{N / 2-1} X(\mathrm{k}) e^{j 2 \pi k t / N}
$$

Where

t- represent time domain and

$\mathrm{K}$ - represent frequency domain

$\mathrm{X}(\mathrm{k})$ is the input signal of particular sample. The above equation shows the Fast Fourier Transform for the particular samples

$$
\mathrm{X}(\mathrm{k})=1 / N \sum_{t=N / 2}^{N / 2-1} x(\mathrm{t}) e^{-j 2 \pi k t / N}
$$

\subsection{FFT (Fast Fourier Transform)}

Decode: sample of time-domain into samples frequencydomain.
Decoding is the procedure in which the adaptation of an encoded arrangement back into the original arrangement of characters. Decoding is used in data infrastructures, networking, and storing.

Orthogonally of any two bins:

$$
\sum_{t=N / 2}^{N / 2-1} e^{-j 2 \pi k t / N} e^{-j 2 \pi p t / N}=0, p \neq k
$$

The orthogonally opinion says that the error vector of the ideal estimator (in a mean squareerror sense) is orthogonal to any conceivable estimator [8]. The orthogonally attitude is most normally stated for linear estimators, but more general preparations are possible. Since the code is a necessary and adequate condition for optimality, it can be used to find the minimum mean square error estimator.

Example; we use modulation scheme\&4 sub-carriers to transmita stream of samples [5].

\begin{tabular}{|c|c|c|c|c|c|c|c|c|c|}
\hline \multirow[b]{3}{*}{ Symbol 1} & \multicolumn{4}{|c|}{ Frequency-domain signal } & \multicolumn{3}{|c|}{ Time-domain signal } & \multirow[b]{3}{*}{0} & \multirow[b]{3}{*}{$2+2 \mathrm{i}$} \\
\hline & c1 & c2 & c3 & c4 & IFFT & & & & \\
\hline & 1 & 1 & -1 & -1 & $\rightarrow$ & 0 & $2-2 i$ & & \\
\hline Symbol 2 & 1 & 1 & 1. & -1 & & 2 & $0-2 \mathrm{i}$ & 2 & $0+2 \mathrm{i}$ \\
\hline Symbol 3 & 1 & -1 & -1 & -1 & & -2 & 0 & 2 & 2. \\
\hline Symbol 4 & -1 & 1 & -1 & -1 & & -2 & $0-2 \mathrm{i}$ & -2 & $0+2 \mathrm{i}$ \\
\hline Symbol 5 & -1 & 1 & 1. & -1 & & 0 & $-2-2 \mathrm{i}$ & 0 & $-2+2 \mathrm{i}$ \\
\hline Symbol 6 & -1 & -1 & 1 & 1. & & 0 & $2+2 i$ & 0 & $-2-2 \mathrm{i}$ \\
\hline
\end{tabular}

$$
1,1,-1,-1,1,1,1,-1,1,-1,-1,-1,-1,-1,-1,-1,1, \ldots
$$

Serial to parallel conversion of samples

- Parallel to serial conversion, \&transmit time domain samples

$0,2-2 \mathrm{i}, 0,2+2 \mathrm{i}, 2,0-2 \mathrm{i}, 2,0+2 \mathrm{i},-2,0,2,2,-2,0-2 \mathrm{i},-2,0-2 \mathrm{i},-2$, $0+2,0,-2-2 \mathrm{i}, 0,-2+2 \mathrm{i}, 0,2+2 \mathrm{i}, 0,-2-2 \mathrm{i}$
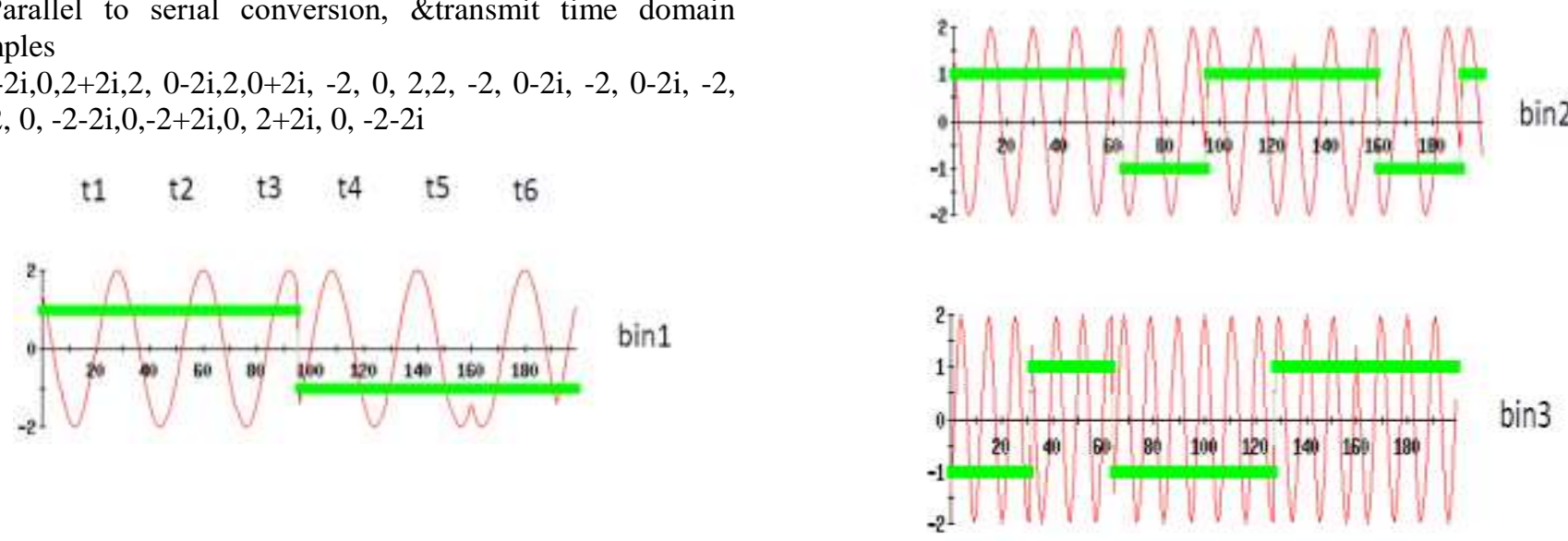


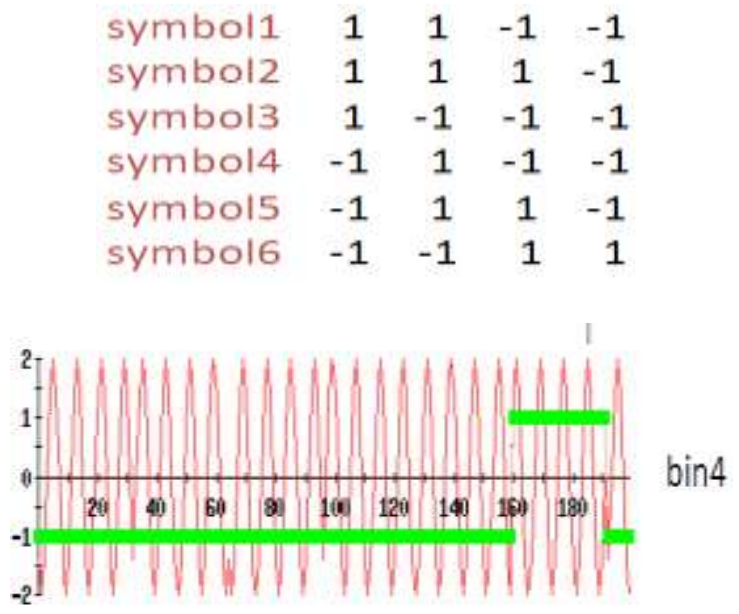

Fig 4: Parallel to serial conversion \& transmit time domain samples [5].

\subsection{Multi-Path Effect}

Fig. 5 shows the multi-path effect. If the pathway from the receiver to the receiver consists of either replications or obstructions, fading possessions, the signal ranges the receiver from many dissimilar routes, reproduction of the unique.

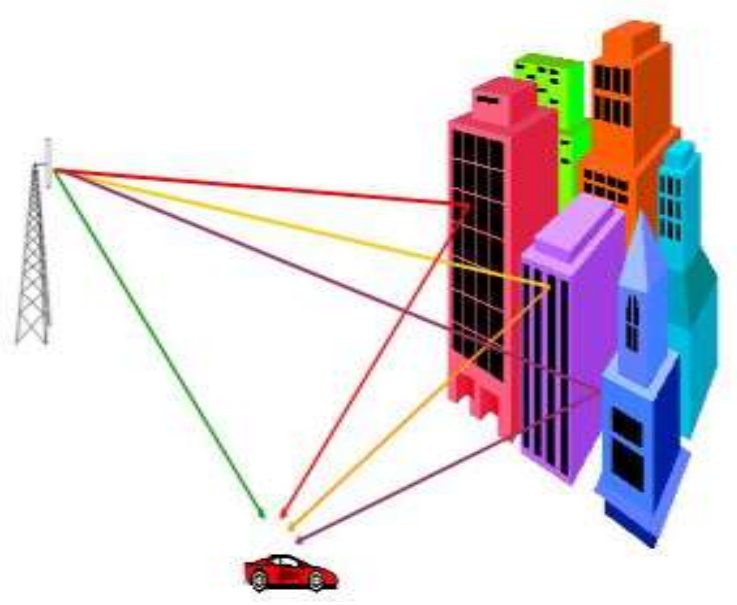

Fig: 5. Multi-path Effect

\subsection{Digital Audio Broadcasting (DAB)}

Digital Audio Broadcasting is a cardinal radio technology for spreading radio stations, used in numerousCountries, exclusively in Europe. DAB is more vigorous with noise and multipath disappearing for mobile communication. It offers additional radio programs over an exact spectrum thananalogyFM radio. In many countries DAB will graduallysubstitute FM radio. Norway announce national FM radio switch off starting from 2017. It has four broadcast modes with different parameters as shown in the Table. 2

Table 2: Digital Audio Broadcasting parameters[7].

\begin{tabular}{|l|l|l|l|l|}
\hline Parameters & Mode I & Mode II & Mode III & Mode IV \\
\hline No. of sub-carriers & 1546 & 383 & 191 & 738 \\
\hline Sub-carrier spacing & $0.5 \mathrm{kHz}$ & $3 \mathrm{kHz}$ & $7 \mathrm{kHz}$ & $2.5 \mathrm{kHz}$ \\
\hline Symbol time & $1.146 \mathrm{~ms}$ & $312.5 \mathrm{us}$ & $165.8 \mathrm{us}$ & $622 \mathrm{us}$ \\
\hline Guard time & $256 \mathrm{us}$ & $62.5 \mathrm{us}$ & $31.8 \mathrm{us}$ & $113 \mathrm{us}$ \\
\hline Carrier frequency & $<365 \mathrm{MHz}$ & $<1.6 \mathrm{GHz}$ & $<2 \mathrm{GHz}$ & $<1.4 \mathrm{GHz}$ \\
\hline $\begin{array}{l}\text { Transmitter } \\
\text { separation }\end{array}$ & $<97 \mathrm{~km}$ & $<23 \mathrm{~km}$ & $<11 \mathrm{~km}$ & $<47 \mathrm{~km}$ \\
\hline
\end{tabular}

The DAB conveyed data contains of number of indications sampled at a rate of $48 \mathrm{kHz}$ with a 22-bit determination [5]. This signal is then crushed at rates extending from 32 to 384 kbps, contingent upon the wanted quality. The subsequent digital data is then alienated into frames of $24 \mathrm{~ms}$. DAB uses discrepancy QPSK variation for the sub-carriers. A valueless symbol indicates the start of the frame. A orientation OFDM symbol is then sent to serve as a preliminary point for the differential deciphering of the QPSK subcarriers. Difference Modulation avoids the use of complicated phase-recovery arrangements. DAB uses a rate quarter convolutional code with a limitation length of 7 for error-correction. Inserting is used to separate the coded bits in the domain frequency as much as possible, which evades large error ruptures in the case of deep fades upsetting a group of sub-carriers.

\section{EXISTING PROBLEM}

The next genration communication system will operate on highly dispersive channel environments in which very dense urban areas added high multipath delay spreads and very high carrier frequencies with high mobile velocities result in high doppler spreads. The OFDM systems rely on frequency band localization waveforms. The high senstivity to doppler spread and frequency synchronization error due to multiple 
access and increased inter carrier and user interference. These systems are significant to out of band emissions and requirement of large guard bands with respect to other adjacent system. The optimization of transmit and receive wave forms for QOS optimization through interference reduction is needed.

\section{RELATED WORK}

An OFDM representation is completed of subcarriers moderated by collections mapping. This planning can be attained from Phase-shift keying (PSK) Quadrature Amplitude Modulation. For an OFDM classification with N sub carriers, the high rapidity two serial input crick is denoted as $\left\{b_{i}\right\}$.

After Serial to Parallel (S-P) convert and collection mapping, a novel Similar Sign Series $\left\{\mathrm{a}_{0}, \mathrm{a}_{1}, \mathrm{a}_{2} \ldots \ldots \ldots\right.$ $\left.\mathrm{a}_{\mathrm{i}}, \ldots \ldots \mathrm{a}_{\mathrm{N}-1}\right\}$ is found, $\mathrm{a}_{\mathrm{i}}$ is a discrete compound valued sign.[6] Here $a_{i} \in\{ \pm\}$ when BPSK planning is accepted When QPSK representing is accepted. When QPSK mapping is used, $\mathrm{a}_{\mathrm{i}} \in\{ \pm 1, \pm i\}$.

Each component of similar signal series issupplied to $\mathrm{N}$ orthogonal

$\left\{e^{j 2 \Pi f 0 t}, e^{j 2 \Pi f 1 t}\right.$ sub-carriers

Lastly moderated signals are extra composed to form an OFDM sign. Use of Discrete Fourier Transformation shortens the OFDM scheme design.

The complex covering of the conveyed OFDM signs can be inscribed as;

$$
y(t)=\frac{1}{\sqrt{ }} \sum_{K=0}^{N-1} Y k e^{j 2 \Pi f k t}, 0 \leq \mathrm{t} \leq N T
$$

Indicators with large $\mathrm{N}$ become Gaussian circulated with Probability Density Utility is given by [7].

$$
\mathrm{P}_{\mathrm{r}}\{\mathrm{y}(\mathrm{t})\}=\frac{1}{\sqrt{2 \pi \sigma}} e^{-\frac{[y(t)] 2}{4 \sigma * \sigma}}
$$

Where $\sigma$ is the variance of $\mathrm{y}(\mathrm{t})$.

PAPR (Peak Average to Power Ratio)is normal, the PAPR of [7] $y(t)$ is the ratio between the maximum simultaneous power and average power;

$$
\operatorname{PAPR}[\mathrm{y}(\mathrm{t})]=\frac{\text { Ppeak }}{\text { Average }}=10 \log _{10} \frac{\max \left[|y(n)|^{2}\right]}{E\left[|y(n)|^{2}\right]} .
$$

Where $\mathrm{P}_{\text {Peak }}$ signifies peak output power $\mathrm{P}_{\text {Average }}$ means average power,E[.] denotes the expected value $\mathrm{y}_{\mathrm{n}}$ characterizes the conveyed OFDM indications which are gotten by taking Inverse Fast Fourier Transformation process on moderated input ciphers, $\mathrm{y}_{\mathrm{n}}$ is expressed as;

$$
\mathrm{Y}_{\mathrm{n}}=\frac{1}{\sqrt{N}} \sum_{K=0}^{N-1} Y_{k} W_{N}^{n k}
$$

Where $W_{N}^{n k}$ is angular speed or frequency

PAPR reduction techniques are therefore of great significance for OFDM systems. Also due to the large instabilities in power output the HPA (high power amplifier) should have large vigorous range. This consequences in deprived power efficiency.

\section{OFDM RESULTS AND EXPLANATION}

The below Fig. 6 shows the Transmission of data in terms of modulation sub-carriers in OFDM technology as the carriers are divided into number of sub carriers and are allocated by the equally spaced bandwidth which will help to modulate the data at higher distances. The Fig. 7 shows the sub carrier indices for the transmitting data for the OFDM for transmitting antenna 1 with the guard bits intervals and pilot sequences. The pilot signals are used for the data synchronization and for the controlling of data in high frequency environments.

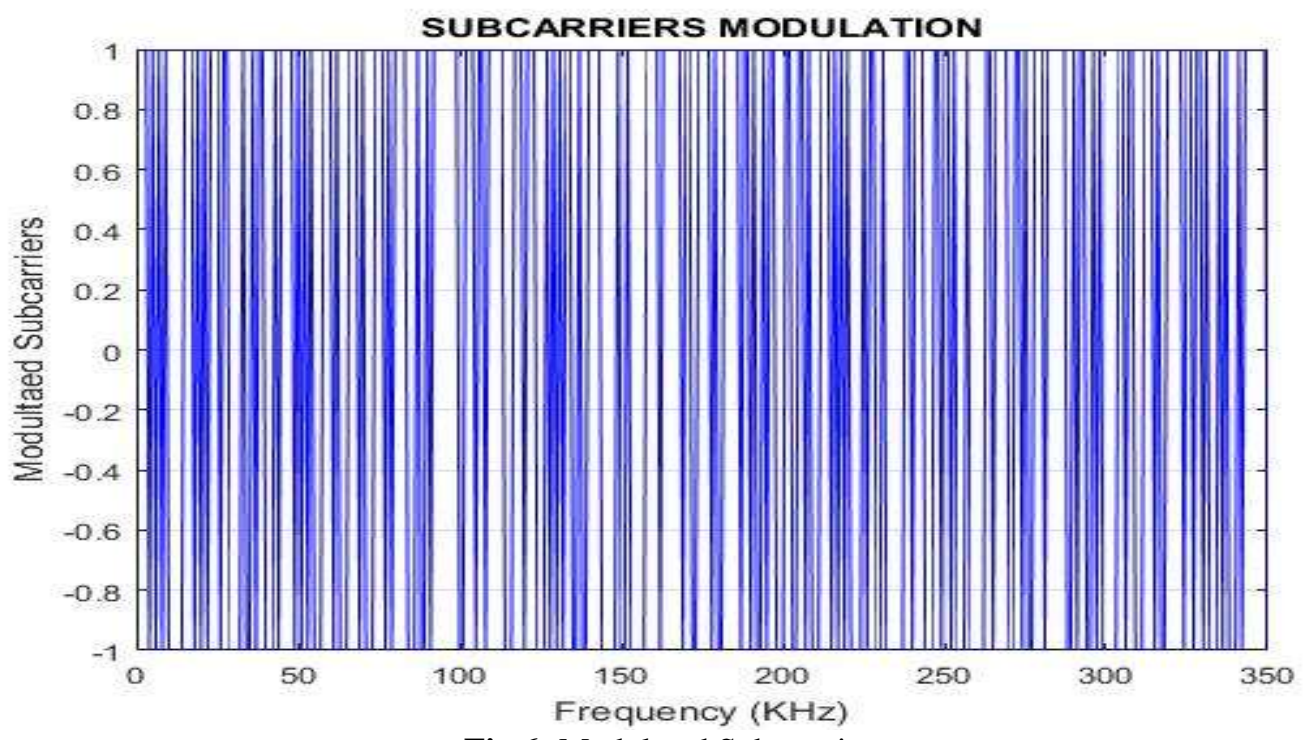

Fig 6: Modulated Sub-carriers 


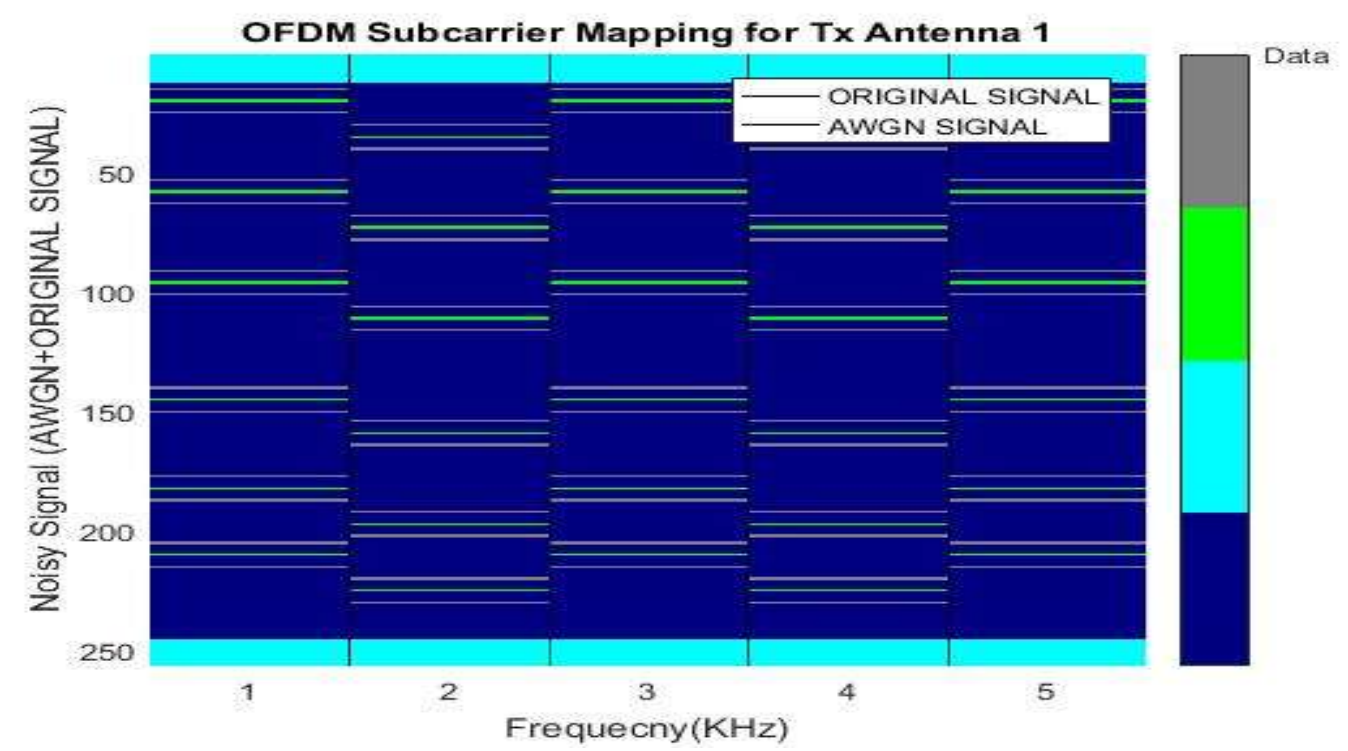

Fig 7: OFDM Sub carrier Mapping for Tx Anteena 1

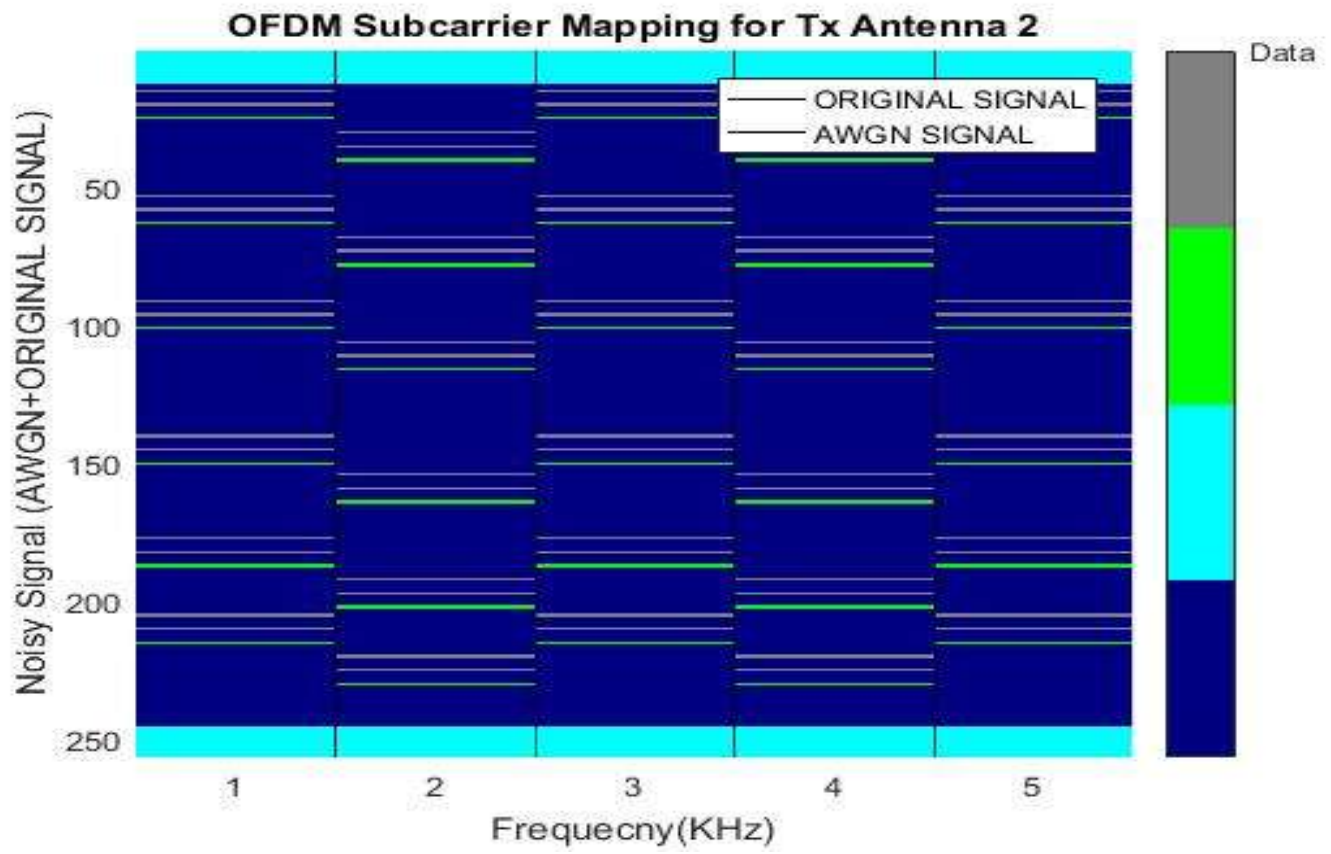

Fig: 8: OFDM subcarrier Mapping for Tx Antenna 2

The Fig. 8 shows the sub carrier indices for the transmitting data for the OFDM for transmitting antenna 2 with the gurad bits intervals and pilot sequences . The Fig.9 shows the mixing of the modulating signal with the additive white Gaussian noise which is responsible to degrade the performance for the evaluation of the Peak to Average ratio. Because at higher distances there are lot of chances for the interference which results in the inter channel interference which will degrade the performance and will results in high PAPR. So the equalization of the signal which will deal with adaptive environment is needed to decrease PAPR. 


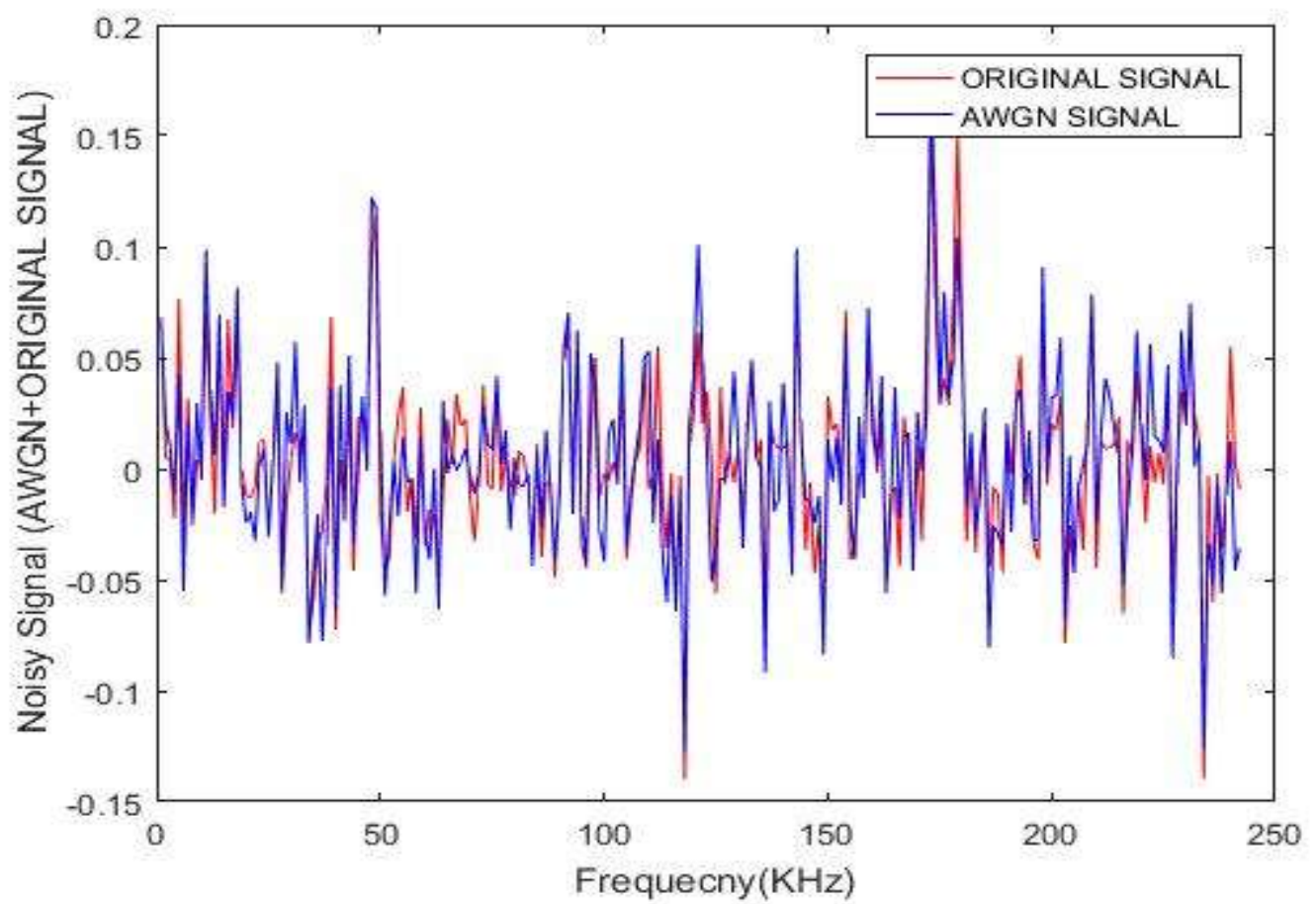

Fig 9:. Noisy Signal (original and AWGN signals)

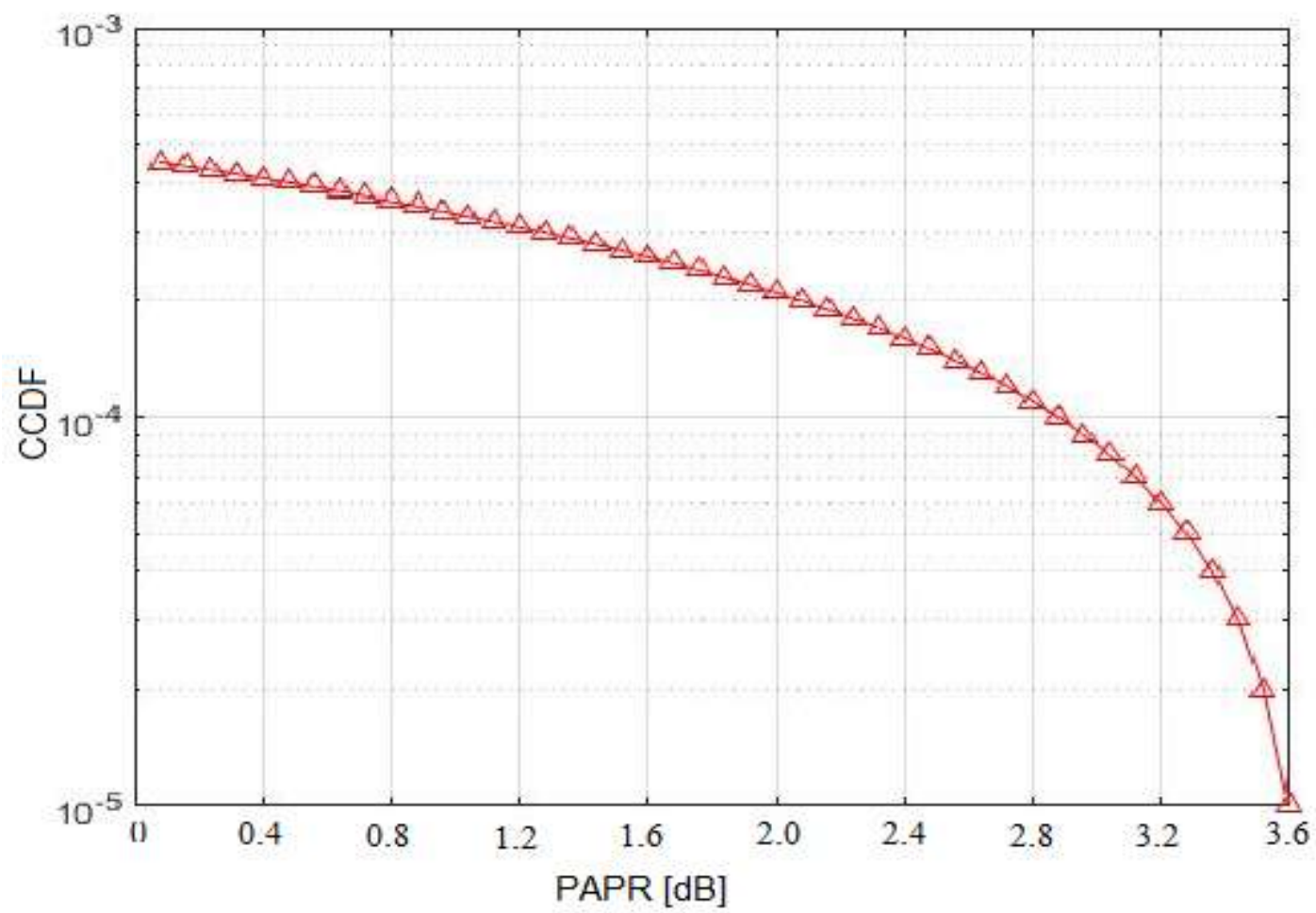

Fig: 10. PAPR (Peak to average power ratio) Reduction graph

The Fig. 10 shows that the performance of the PAPR is decreasing which shows that our proposed approach named as pulse shaping and equalization process is giving efficient results and will help to decrease the interference levels using OFDM technology. By using pulse shaping and equalization PAPR reduced from $7.5 \mathrm{~dB}$ to $3.6 \mathrm{~dB}$.
Table: 3 Comparison between PAPR (previous and Proposed Work)

\begin{tabular}{|l|l|}
\hline $\begin{array}{l}\text { Performance } \\
\text { Parameters }\end{array}$ & PAPR \\
\hline Proposed Work & $3.6 \mathrm{~dB}$ \\
\hline Previous Work & $7.5 \mathrm{~dB}$ \\
\hline
\end{tabular}




\section{CONCLUSION AND FUTURE SCOPE}

From the proposed approach we have conclude that the system will be able to reduce the PAPR effect which is responsible in reducing the inter symbol interference that will degrade the performance of the OFDM systems. So our shaping pulse and equalization approach will reduce this effect and the sub carriers with equally spaced bandwidth will achieve high throughput at the receiver end and shows that the effect of white Gaussian noise doesn't affect much using our proposed scheme and is highly reliable and scalable at the receiver end. Future studies on PAPR reduction may contain a grouping of different schemes. The practical execution studies will consider the non-linearity effects of the optoelectronic strategies that may limit the modulation order. In future scope we can implement the hybrid approach of the Pulse shaping and Match Filtering methods reduce the PAPR and Inter symbol intrusion performance parameters.

\section{REFERENCES}

[1] Han, SeungHee, and Jae Hong Lee. "An overview of peak-to-average power ratio reduction techniques for multicarrier transmission." Wireless Communications, IEEE 12.2 (2005): 56-65.

[2] Haene, Simon, David Perels, and Andreas Burg. "A real-time 4-stream MIMO-OFDM transceiver: system design, FPGA implementation, and characterization." IEEE Journal on Selected Areas in Communications 26.6 (2008): 877-889.

[3] Adve, Raviraj S., and Tapan K. Sarkar. "Simultaneous time-and frequency-domain extrapolation." IEEE Transactions on

[4] Tarighat, Alireza, Rahim Bagheri, and Ali H. Sayed. "Compensation schemes and performance analysis of IQ imbalances in OFDM receivers." IEEE Transactions on Signal Processing 53.8 (2005): 32573268.Robertson, Patrick, and Stefan Kaiser.

[5] "Analysis of the effects of phase-noise in orthogonal frequency division multiplex (OFDM) systems."Communications, $1995 . \quad$ ICC'95 Seattle,'Gateway to Globalization', 1995 IEEE International Conference on. Vol. 3. IEEE, 1995.

[6] Cimini, Leonard. "Analysis and simulation of a digital mobile channel using orthogonal frequency division multiplexing." IEEE transactions on communications 33.7 (1985): 665-675.

[7] Jayalath, A. D. S. and C. Tellambura, "Use of data permutation to reduce the peak-to-average power.

[8] Gross, R. and D. Veeneman, "Clipping distortion, in DMT ADSL systems," IEEE Electron. Lett., Vol. 29, 2080-2081, Nov. 1993.

[9] Wu, Yiyan, and William Y. Zou. "Orthogonal frequency division multiplexing: a multi-carrier modulation scheme." IEEE Transactions on Consumer Electronics 41.3 (1995): 392-399.

[10] Zhidkov, Sergey V. "Performance analysis and optimization of OFDM receiver with blanking nonlinearity in impulsive noise environment." IEEE transactions on vehicular technology 55.1 (2006): 234242.

[11] Lee, King F., and Douglas B. Williams. "A spacefrequency transmitter diversity technique for OFDM systems." Global Telecommunications Conference, 2000. GLOBECOM'00. IEEE. Vol. 3. IEEE, 2000.

\section{BIOGRAPHIES}

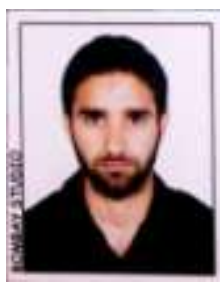

Rizwan Ahmed Malik is presently pursuing Masters of Technology from Chandigarh Engineering College, Landran in the field of electronics and communication. He received his Bachelor's degree in 2014 from BGSBU, Rajouri (j\&k).

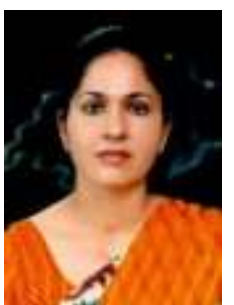

Dr. Ruchi Singla is presently working as HOD in ECE department at Chandigarh Engineering College, Landran. She has presented/published a number of papers in national and international conferences/journals 